\title{
Thermonuclear combustion in steep density gradients.
}

\author{
Camille Charignon* \\ CEA, IRFU, SAp, 91191 Gif-sur-Yvette, France \\ E-mail: camille.charignondcea.fr \\ Jean-Pierre Chièze \\ CEA, IRFU, SAp, 91191 Gif-sur-Yvette, France \\ E-mail: [chiezedcea.fr]
}

\begin{abstract}
We study a new mechanism for deflagration to detonation transition in SN Ia, based on the formation of shocks by amplification of sound waves in the steep density gradients of white dwarfs. We characterize, in terms of wavelength and amplitude, the perturbations which will ignite a detonation after amplification. We found the critical parameter to be the height of shock formation. When it occurs in the inner dense regions $\left(\rho>10^{6} \mathrm{~g} \mathrm{~cm}^{-3}\right)$, detonation is inevitable but can take an arbitrarily long time. On the contrary no ignition occurs when shocks initiated by small amplitude or large wavelength perturbations form further away in less dense regions. We consider here a spherical self-gravitating model of a Chandrasekhar mass $\mathrm{C}+\mathrm{O}$ white dwarf, with a steep density gradient near the envelope. For equimass $\mathrm{C}+\mathrm{O}$ composition, detonation initiation requires a $2 \%$ velocity perturbation level corresponding to a Mach number $\mathrm{M}=0.02$. Small perturbations $(\mathrm{M} \sim 0.003)$ could however trigger detonations if a thin outer helium layer is considered.
\end{abstract}

XII International Symposium on Nuclei in the Cosmos

August 5-12, 2012

Cairns, Australia

${ }^{*}$ Speaker. 


\section{Introduction}

Type Ia supernovae are thought to result from the thermonuclear explosion of a carbon oxygen white dwarf. Presently, two main families of models are proposed: in the single degenerate scenario []] explosion is triggered by accretion of hydrogen or helium from a non-degenerate companion until Chandrasekhar mass $\left(M_{c h}\right)$ is reached; in the double degenerate scenario [B] the explosion results from the merging of two $\mathrm{C}+\mathrm{O}$ white dwarfs. In the latter mechanism, only the detonation combustion mode can release enough energy to prevent a collapse and produce a healthy explosion. Furthermore, detonation ignition is not so easily achieved and requires a violent merger [ए]], which might not be frequent enough to explain the SN Ia rates. In the former scenario, pure detonation and pure deflagration fail to reproduce light curves and nucleosynthesis. Detonation models incinerate the whole star at high density producing mostly ${ }^{56} \mathrm{Ni}$ and resulting in wrong nucleosynthesis. On the other hand, a deflagration is very subsonic and gives time for expansion so that burning also occurs at lower density and produces the intermediate elements needed for spectra. But pure deflagration models are under-energetics [ [] . Accordingly, the delayed detonation scenario has been proposed [团] to combine the advantages of both combustion modes. In this model, a flame born as a subsonic deflagration is accelerated by turbulence and turns at some point into a detonation. The initial phase allows the star to expand so that intermediate mass elements are produced in the final detonation stage.

However, despite extensive studies, the mechanism of deflagration-to-detonation transition (DDT) is still unclear. It has been shown by 3D simulations [6] that this mechanism was the most promising path for single degenerate models to reasonably account for observables. However, due to the lack of theoretical understanding of this transition, the turning point between deflagration and detonation is still a free parameter. On earth, DDT has been observed in confined media, for which the transition seems to occur through shock-flame interactions, which are due to shock reflections on obstacles or walls. In unconfined media, it has been proposed that the transition should occur through the gradient mechanism [5], where a suitable induction time gradient is created and maintained over a time long enough for a coherent burning wave to build up pressure and create a self-sustained detonation. It has been suggested that the turning point from turbulent deflagration to detonation might be the creation of such a flat gradient at the transition between the flamelet and the distributed combustion regimes, where the flame changes in nature. However it is not clear if the turbulence level necessary for the distributed regime to occur is achieved during the explosion, even at the late stages. More recently, through numerical studies [Q] claimed that intense enough turbulence could directly initiate DDT, without relying on this induction time gradient. Thus, in the context of SN Ia where the plasma is fully unconfined but where the flame is highly turbulent, the possibility of DDT is still debated.

Here, we examine a new mechanism in which pressure perturbations, created by flame turbulence, are amplified during their propagation ahead of the flame in the steep density gradient. Thus when they turn into shocks, they can be strong enough to ignite a detonation. Turbulence alone can produce pressure perturbations, thus turbulent combustion is likely to be a powerful source of perturbations. Sound waves propagating through steep density gradients get amplified and with a large enough density decrease, strong shocks form and provide substantial heating. The mechanism of formation of strong shocks in a density gradient has been proposed, for example, as a possible 
heating source for the solar chromosphere (see [W] for a review). We then analyse the propagation properties of finite amplitude pressure waves in $\mathrm{C}+\mathrm{O}$ degenerate matter, in a spherical WD model. We determine the frequencies and amplitudes which lead to a successful delayed detonation. Finally we briefly discuss the applicability of this mechanism to thermonuclear supernovae.

\section{Perturbation amplification and ignition conditions}

This ignition mechanism is based on the amplification of small perturbations in the steep density gradient of white dwarfs. A perturbation of velocity amplitude $u$ in a fluid of density $\rho$ with sound velocity $C_{s}$, carries a momentum flux $F=\frac{1}{2} \rho u^{2} C_{s}$. In spherical geometry, since the surface of the spherical wave over which the initial momentum is distributed is proportional to $r^{2}$ the conservation of momentum reads:

$$
\rho u^{2} C_{s} 4 \pi r^{2}=\rho_{0} u_{0}^{2} C_{s, 0} 4 \pi r_{0}^{2},
$$

with $\rho_{0}, C_{s, 0}$ and $u_{0}$ the density, sound speed and velocity amplitude at the position $r_{0}$ of the perturbation emitter. This gives for the velocity perturbation at any radii:

$$
u(r)=u_{0} \sqrt{\frac{\rho_{0} C_{s, 0}}{\rho(r) C_{s}(r)}} \frac{r_{0}}{r} .
$$

In degenerate matter, sound speed depends mainly on the density. Thus, a wave travelling outward will slow down, so that the perturbation amplitude will grow to conserve the flux. Except in the inner region where the geometrical damping $\left(r_{0} / r\right)$ can be stronger than the amplification factor $\sqrt{\rho_{0} C_{s, 0} / \rho(r) C_{s}(r)}$. The maximum of $\rho\left(r_{0}\right) C_{s}\left(r_{0}\right) r_{0}^{2}$ is the optimum position between these two opposite effects. It lies around $350 \mathrm{~km}$ from the center for the cold $M_{c h}$ white dwarf considered here. For this structure, these competing effects result in overall amplification after a possible damping in the central region. As it propagates, a finite amplitude wave always steepens and eventually forms a shock. But due to amplification, according to equation ([2.]), the shock will be formed with higher amplitude. After formation, kinetic energy dissipation inhibits further growth. The radius of shock formation, $r_{s h}$, corresponds to the position where the hills of the wave catch up the valleys. The hills move with respect to the wave at $+u(r)$ while the valleys at $-u(r)$ and they merge when they have travelled their initial separation of half the initial wavelength: $\lambda_{0} / 2$. For a perturbation initiated at $r_{0}$, this reads:

$$
\int_{r_{0}}^{r_{s h}} u_{0} \sqrt{\frac{\rho_{0} C_{s, 0}}{\rho C_{s}}} \frac{d r}{C_{s}}=\frac{\lambda_{0}}{4},
$$

This formula provides a quite good estimate of the radius at which a perturbation turns into a shock and starts to dissipate and heat up the medium. If $r_{s h}$ is small enough so that shock heating takes place in regions dense enough for detonation ignition, the transition is inevitable but may require a large number of shocks. When $r_{s h}$ is large, so that $\rho\left(r_{s h}\right)<10^{6} \mathrm{~g} \mathrm{~cm}^{-3}$, ignition is impossible whatever the number of shocks.

The case of an initial perturbation of $500 \mathrm{~km} \mathrm{~s}^{-1}(M \sim 0.05)$ originating close to the centre $\left(r_{0}=100 \mathrm{~km}\right)$ is shown on Fig.W. The simulation, in blue, is compared to the predictions of formula 

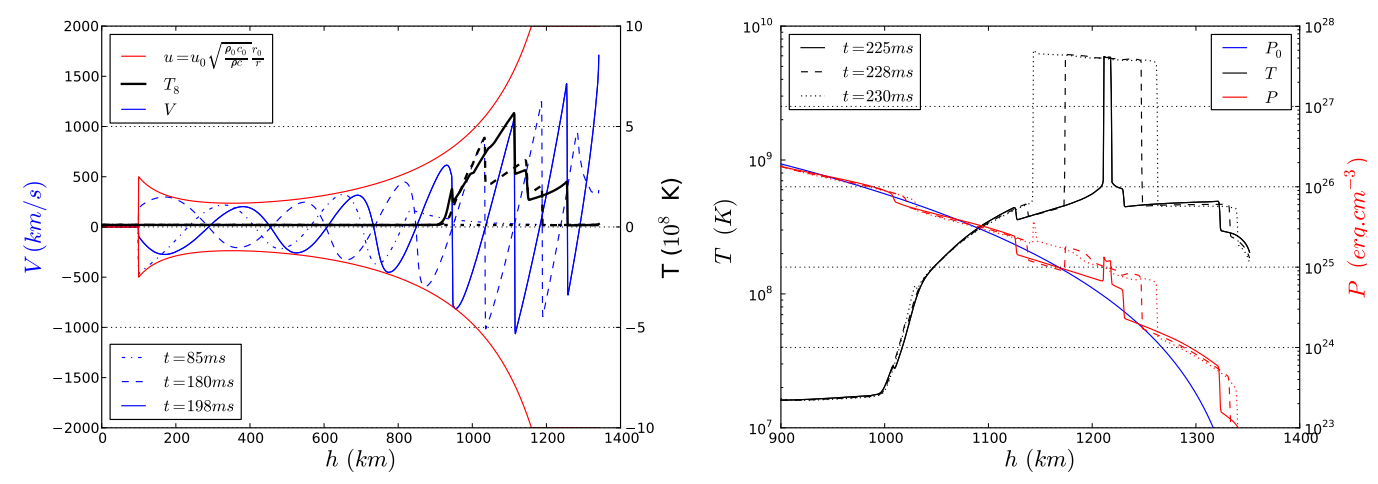

Figure 1: On the left panel, we show the propagation of a $500 \mathrm{~km} \cdot \mathrm{s}^{-1}$ perturbation of period $36 \mathrm{~ms}$ initiated at a $r_{0}=100 \mathrm{~km}$. The period corresponds to a wavelength of $\lambda_{0}=0.25 R_{W D}$ at the optimal radius: $r_{0}=350 \mathrm{~km}$. We compare the theoretical formula (2.1) in red to the simulation in blue. On the right panel the ensuing detonation ignition and propagation is shown. The pressure in red and the temperature in black are plotted at three subsequent times.

(2.]) in red. Initially, when the curvature effect is strong, damping dominates the amplification because of the relatively flat density gradient in the central region, and the perturbation amplitude cannot grow. Then, the gradient steepens while the effect of growing wave surface weakens, and the waves are amplified. The transition to shock occurs at a height of $1100 \mathrm{~km}$. There the velocity of the simulation diverges from the prediction, due to dissipation of the shock kinetic energy. At that point the wave begins to heat up the plasma. After a few shocks, the ignition temperature is reached. The ensuing thermonuclear runaway creates a strong overpressure sufficient to launch a double detonation both upwards and downwards.

We now turn to the results of our parametric study. The number of shocks needed to ignite a detonation is presented in table 1 , for 4 different periods $\left(T_{\text {osc }}\right)$ and amplitudes varying from 1000 to $100 \mathrm{~km} \mathrm{~s}^{-1}$, corresponding to Mach number of 0.1 to 0.01 . We observe two trends. First, the larger the amplitude the stronger the shock, leading to faster detonation ignition. Also smaller amplitude turns to shock further away (eq.2.2) and below a certain threshold it is formed in regions with densities too low to ignite a detonation. Second, the smaller the period the weaker the shock due to earlier shock formation (eq. 2.2 ) and dissipation. Thus, more shocks are needed for ignition. For the pure $\mathrm{C}+\mathrm{O}$ case, a quite large initial perturbation is needed: $u_{0}=200 \mathrm{~km} \cdot \mathrm{s}^{-1}$, corresponding to a Mach number of $M_{0}=0.02$. We show in the following that the initial perturbation may be reduced to $30 \mathrm{~km} \cdot \mathrm{s}^{-1}$ or $M_{0}=0.003$ by the presence of a helium layer.

Helium layer: This layer at the top of the $\mathrm{C}+\mathrm{O}$ core favours a detonation. It should be small in order to keep the observables undisturbed. The presence of a helium shell above the $\mathrm{C}+\mathrm{O}$ core would lead to unobserved peculiar light curves and spectra if the shell is larger than $\sim 0.05 M_{\odot}$. Accordingly, we considered a very thin helium layer of $M_{H e}=0.01 M_{\odot}$. Since helium combustion is strongly exothermic this layer detonates with weaker shocks. Once ignited, these He-detonations are strong enough to initiate $\mathrm{C}+\mathrm{O}$-detonation when they reach the $\mathrm{C}+\mathrm{O}$ core. The set-up here is different from the double detonation scenario, where an accretion induced detonation is initiated 


\begin{tabular}{|c|c|c|c|c|c|c|}
\hline$\delta_{u}\left(\mathrm{~km} \cdot \mathrm{s}^{-1}\right)$ & 1000 & 500 & 400 & 300 & 200 & 100 \\
\hline$T_{\text {osc }}=72 \mathrm{~ms}$ & 1 & 2 & 3 & 4 & - & - \\
$T_{\text {osc }}=36 \mathrm{~ms}$ & 2 & 5 & 7 & 10 & - & - \\
$T_{\text {osc }}=18 \mathrm{~ms}$ & 4 & 13 & 17 & 34 & 67 & - \\
\hline$+M_{H e}=0.01 M_{\odot}$ & \multicolumn{7}{|l|}{} \\
\hline$\delta_{u}\left(\mathrm{~km} \cdot \mathrm{s}^{-1}\right)$ & 1000 & 500 & 250 & 100 & 50 & 25 \\
\hline$T_{\text {osc }}=72 \mathrm{~ms}$ & 1 & 1 & 1 & 1 & - & - \\
$T_{\text {osc }}=36 \mathrm{~ms}$ & 2 & 1 & 1 & 3 & 5 & 20 \\
$T_{\text {osc }}=18 \mathrm{~ms}$ & 4 & 5 & 6 & 9 & 19 & 78 \\
$T_{\text {osc }}=10 \mathrm{~ms}$ & 7 & 34 & 35 & 47 & & \\
\hline
\end{tabular}

Table 1: The number of shocks needed to ignite a detonation are shown for acoustic perturbations of different periods $\left(T_{o s c}\right)$ and initial velocity amplitudes $\left(u_{0}\right)$, propagating in a pure $\mathrm{C}+\mathrm{O}$ white dwarf (upper table), or in a $\mathrm{C}+\mathrm{O}$ white dwarf with a $0.01 M_{\odot}$ helium layer (lower table). Perturbations are initiated at the optimal radius $\left(r_{0}=350 \mathrm{~km}\right)$. The period $T_{o s c}=72 \mathrm{~ms}$ corresponds to an initial wavelength of $\lambda_{0}=0.5 R_{W D}$ at this radius.

at the base of the helium layer. In that case, the transmission of the detonation to the core may need to wait for the He-detonation induced shocks to convergence inside the core and ignite the carbon. Indeed helium ignites either above the core/envelope boundary - which leaves time for the inward propagating detonation to build up large enough overpressure to directly trigger the $\mathrm{C}+\mathrm{O}$-detonation - or at the transition with sufficiently compressed and heated carbon to produce an outward moving He-detonation and an inward moving $\mathrm{C}+\mathrm{O}$-detonation. And even if this fails, the usually invoked shock convergence [8] could still trigger the $\mathrm{C}+\mathrm{O}$-detonation, with some additional

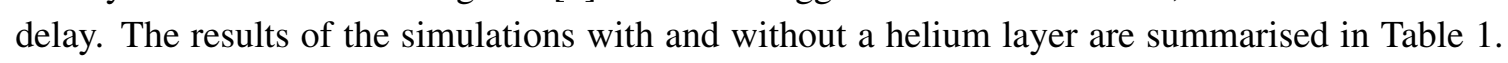
Clearly, the presence of a helium layer favours ignition by weak perturbations, down to $25 \mathrm{~km} \mathrm{~s}^{-1}$ or $M_{0} \sim 0.003$.

\section{Discussion}

Finally, we can estimate the energy flux, $F_{\text {det }}$, needed for a cold white dwarf to detonate through this mechanism and compare it to an estimate of the energy flux, $F_{n u c}$, generated by the thermonuclear flame of velocity $V_{f l}$ during the initial deflagration phase:

$$
\begin{gathered}
F_{d e t}=0.5 \rho_{0} C_{s, 0} u^{2} \sim 6.510^{31} \mathrm{erg} . \mathrm{cm}^{-2} \mathrm{~s}^{-1}, \\
F_{n u c}=V_{f l} \rho \varepsilon_{n u c}=7.610^{33} \mathrm{erg} . \mathrm{cm}^{-2} \mathrm{~s}^{-1} .
\end{gathered}
$$

We have assumed a density of $\rho_{0}=10^{9} \mathrm{~g} . \mathrm{cm}^{-3}$ and used an estimated laminar flame velocity of $V_{f l}\left(\rho_{0}=10^{9}\right)=30 \mathrm{~km} / \mathrm{s}$ and a nuclear energy release $\varepsilon_{\text {nuс }}=3.810^{17} \mathrm{erg} \mathrm{cm}^{-3}$, corresponding to the combustion of $X_{O}=X_{C}=0.5$. The laminar deflagration speed represents here a lower limit since it is greatly enhanced by turbulence. But even in this limit, injecting only $1 \%$ of the generated energy into sound waves would be sufficient for a successful ignition. The time scale over which a transition to detonation has to occur poses a strong constraint on perturbations frequencies and 
amplitudes. In the context of the delayed detonation model of SNe Ia, the detonation has to be triggered after an initial phase of expansion due to the energy released by the deflagration. In the classical local DDT, many studies agree that the detonation has to occur when the flame reaches densities of about $10^{7} \mathrm{~g} \mathrm{~cm}^{-3}$. The DDT mechanism proposed here is actually non local, with a spatial shift between the deflagration producing the perturbations and the position where they trigger a detonation. Thus, the above critical density, usually derived from the transition to the flame distributed regime, is not directly relevant to the present mechanism.

Finally, there are two kinds of perturbations that could successfully trigger a non-local DDT, waves with large wavelengths and small amplitudes or conversely, with small wavelengths and large amplitudes. The former case corresponds to small perturbations over a sizeable fraction of the gradient scale height, for example collisions of Rayleigh-Taylor mushrooms. The latter correspond to repeated strong pressure perturbations, which could be associated with turbulent combustion. In fact, for the standard gradient mechanism DDT [5], pressure perturbations have to build up directly to detonation pressure, and this requires to travel more than $10 \mathrm{~km}$. In the present case, a weak build up to only a fraction of the detonation pressure would be enough if sufficiently repeated. Moreover, in an attempt to get direct transition to detonation, Poludnenko [Q] obtained repeated (every eddy turn-over time) strong pressure pulses of about $30 \%$ of the unperturbed pressure. We propose a promising possibility for failed local DDT to revive as a non-local DDT, after further amplification along the density gradient.

\section{References}

[1] P. Ulmschneider, On Frequency and Strength of Shock Waves in the Solar Atmosphere, Sol. Phys. 12:403-415, 1975.

[2] J. Whelan and I. Iben, Binaries and Supernovae of Type I, ApJ 186:1007-1014, 1973.

[3] I. Iben and A. V. Tutukov, Supernovae of type I as end products of the evolution of binaries with components of moderate initial mass, ApJS 54:335-372, 1984.

[4] A. M. Khokhlov, Delayed detonation model for type IA supernovae, A\&A 245:114-128, 1991.

[5] A. M. Khokhlov, Mechanisms for the initiation of detonations in the degenerate matter of supernovae, A\&A 246:383-396, 1991

[6] V. N. Gamezo, A. M. Khokhlov and E. S. Oran, Three-dimensional Delayed-Detonation Model of Type Ia Supernovae, ApJ 623:337-346, 2005

[7] F. K. Röpke, W. Hillebrandt, W. Schmidt, J. C. Niemeyer, S. I. Blinnikov, and P. A. Mazzali, A Three-Dimensional Deflagration Model for Type Ia Supernovae Compared with Observations, ApJ 668:1132-1139, 2007

[8] M. Fink, F. K. Röpke, W. Hillebrandt, I. R. Seitenzahl, S. A. Sim, and M. Kromer, Double-detonation sub-Chandrasekhar supernovae: can minimum helium shell masses detonate the core?, A\&A 514:A53, 2010

[9] A. Y. Poludnenko, T. A. Gardiner, and E. S. Oran, Spontaneous Transition of Turbulent Flames to Detonations in Unconfined Media, Phyical Review Letters 107(5)054501, 2011

[10] R. Pakmor, M. Kromer, S. Taubenberger, S. A. Sim, F. K. Röpke, and W. Hillebrandt, Normal Type Ia Supernovae from Violent Mergers of White Dwarf Binaries, ApJ 747:L10, 2012 